\title{
ON THE CARDINALITY OF SOLUTIONS OF MULTILINEAR DIFFERENTIAL EQUATIONS AND APPLICATIONS
}

\author{
IOANNIS K. ARGYROS \\ Department of Mathematics \\ University of Iowa \\ Iowa City, Iowa 52242 U.S.A. \\ (Received October 18, 1985)
}

ABSTRACT. We study the existnece and cardinality of solutions of multilinear differential equations giving upper bounds on the number of solutions.

KEY WORDS AND PHRASES. Cardinality, multilinear, bound, beams. 1980 AMS SUBJECT CLASSIFICATION COSE. 34A20, $46 \mathrm{~B} 15$.

1. INTRODUCTION.

Let $n(i), i=1,2, \ldots, m$ be positive integers such that $n(1) \geq n(2) \geq \ldots \geq n(m)$ and let $L_{i}=\sum_{j=0}^{n(i)} C_{i j} D^{j}, i=1,2, \ldots, m$ be regular linear differential operators defined on $c^{n(I)}(I)$, where $I=[a, b]$ usually (but not necessarily). The coefficient functions $C_{i j}, i=1,2, \ldots, m, j=0,1,2, \ldots, n(i)$ are never vanishing real and continuous on $I$.

Using some ideas from [I] and [3] we study the branching of solutions $u \in C^{n(1)}(I)$ to the multilinear equation

$$
M u=\left(L_{1} u\right)\left(I_{2} u\right) \ldots\left(I_{m} u\right)=0
$$

Equation (1.1) is related with the null set $N(M)$

$$
N(M)=\left\{u \in C^{n(I)}(I): M u=0\right\}
$$

which can be infinite dimensional.

We give necessary and sufficient conditions for a $(m-1)$-tuple $\left(\alpha_{1}, \alpha_{2}, \ldots, \alpha_{m-1}\right)$ to be a multiple ordinary branching of a solution to (l.1) where $\alpha_{e} \in I$, $\mathrm{e}=1,2, \ldots, \mathrm{m}-1$.

We also study the existence and cardinality of solutions to the initial value problem

$$
D^{n(1)} u(z)=z_{i}, i=1,2, \ldots, n(1)-1
$$

where $z, z_{i} \in I$, giving upper bounds on the number of solutions with $n$ multiple branchings.

Multilinear equations have a rather extensive literature [3], [4], [6]. A few 
special cases of applications (e.g., pursuit problems and bending of beams) may be formulated in the form $(1,1)$.

Finally we study the problem

$$
\frac{d M}{d \lambda}-\lambda M=0
$$

when it assumes the form (1.I) for some function $\lambda$.

2. BASIC THEOREMS.

DEFINITION 1. Let $B_{1}, B_{2}, \ldots$ and $B_{m}$ denote bases for $N\left(L_{1}\right), N\left(I_{2}\right), \ldots$ and $\mathrm{N}\left(\mathrm{L}_{\mathrm{m}}\right)$ respectively where

$$
B_{i}=\left\{U_{1 i}, U_{2 i}, \ldots, U_{n(i) i}\right\} \text { with } \operatorname{dim}\left(B_{i}\right)=n(i), i=1,2, \ldots, m
$$

and let

$$
E_{j}=\left(B_{j} \cap C^{n(I)}(I)\right)-B_{j-I} \text { with } \operatorname{dim}\left(E_{j}\right)=\bar{n}(j)<n(j), j=2,3, \ldots, m .
$$

Obviously $N\left(L_{1}\right) \cup N\left(L_{2}\right) \cup \ldots \cup N\left(L_{m}\right) \subset N(M)$. We will seek solutions $u \in N(M)$ of the form

$$
u_{\alpha_{1} \alpha_{2} \ldots \alpha_{m-1}}(x)=u(x)=\left\{\begin{array}{lr}
\sum_{j=1}^{n(1)} c_{1 j} u_{1 j}(x)=u_{\alpha_{1}}(x) & \alpha \leq x \leq \alpha_{1} \\
\vdots & \alpha_{e-1} \leq x \leq \alpha_{e} \\
\sum_{j=1}^{n(e)} c_{e j} u_{e j} & \alpha_{e} \leq x \leq \alpha_{e+1} \\
\sum_{j=1}^{n(e+1)} c_{e+1 j} u_{e+1 j} & \alpha_{m-1} \leq x \leq b \\
\vdots \\
\sum_{j=1}^{n(m)} c_{m j} u_{m j}=u_{\alpha_{m}}(x)
\end{array}(2.2)\right.
$$

for $\alpha_{e} \in I, e=1,2, \ldots, m-1$ and $\alpha_{e} \notin N\left(L_{e}\right) \cup N\left(L_{e+1}\right)$. A function of the form (2.I) in $N(M)$ will be said to have a single ordinary branching at $x=\alpha_{e}$, on $\left[\alpha_{e-1}, \alpha_{e+1}\right]$. A function of the form (2.2) will be said to have a multiple ordinary branching at $\left(\alpha_{1}, \alpha_{2}, \ldots, \alpha_{m-1}\right)$ on $I=[a, b]$ with $\alpha_{e} \leq \alpha_{e+1}, e=1, \ldots, m-2$.

Denote the Wronskian

by

$$
w_{e}\left(u_{1 i}, u_{2 i}, \ldots, u_{n(i) i}, u_{1(i+1)}\left(x_{0}\right)\right.
$$

$$
w_{e}\left(x_{0}\right), e=1,2, \ldots, m-1 \text {. }
$$

The following theorem shows when $N(M)$ will contain functions having a multiple ordinary branching.

THEOREM 1. Assume that

$$
n(e)-\bar{n}(e)+n(e+1) \geq n(1)+1, e=2, \ldots, m-1
$$

and if

(i) $E_{j}$ has just one function $u_{1 j}(x), j=2, \ldots, m$, then there exists $u \in N(M)$ having a multiple ordinary branching at $\left(\alpha_{1}, \ldots, \alpha_{m-1}\right)$ if and only if

$$
\dot{w}_{e}\left(\alpha_{e}\right)=0, e=1, \ldots, m-1 \Leftrightarrow\left(L_{i} u_{1}(i+1)\left(\alpha_{i}\right)=0, i=1, \ldots, m-1 .\right.
$$


(ii) $\operatorname{dim}\left(E_{j}\right) \neq l, j=2, \ldots, m$, then for every $\left(a_{1}, \ldots, a_{m-1}\right)$ with $\alpha_{e} \in$ int $I$, and $a_{e} \geq a_{e+1}, e=1, \ldots, m-2$ there exists a $u \in N(M)$ having a multiple ordinary branching at $\left(a_{1}, \alpha_{2}, \ldots, \alpha_{m-1}\right)$.

PROOF. It is enough to find numbers, $c_{11}, \ldots, c_{1 n(1)}, c_{21}, \ldots c_{2 n(2)}, \ldots c_{m l}$, $\ldots, C_{m n(m)}$, so that $u \in C^{n(I)}(I)$. Therefore we must have

$$
\begin{aligned}
& \sum_{j=1}^{n(1)} c_{1 j} u_{1 j}^{(k)}\left(\alpha_{1}\right) \quad=\sum_{j=1}^{\bar{n}(2)} c_{2 j} u_{2 j}{ }^{(k)}\left(\alpha_{1}\right) \\
& \sum_{j=1}^{n(2)} c_{2 j} u_{2 j}{ }^{(k)}\left(\alpha_{2}\right)=\sum_{j=1}^{\bar{n}(3)} c_{3 j^{u} u^{(k)}}\left(\alpha_{2}\right), k=0,1, \ldots, n(1) \\
& \sum_{j=1}^{n(m-1)} c_{m-1 j} u_{m-1 j}^{(k)}(x)=\sum_{j=1}^{\bar{n}(m)} c_{m j} u_{m j}(k)\left(\alpha_{m-1}\right)
\end{aligned}
$$

CASE (i). In this case (2.5) becomes

$$
\sum_{j=1}^{n(e)} c_{e j} u_{e j}{ }^{(k)}\left(\alpha_{e}\right)-c_{1}^{n(e+1)} u_{1 n(e+1)}\left(\alpha_{e}\right)=0, e=1,2, \ldots, m-1, k=0,1, \ldots, n(1)
$$

where $c_{\ln (e+1)} \neq 0$ (we take $c_{\ln (e+1)}=1$ ). The homogeneous equation (2.6) has a nontrivial solution if and only if $(2.4)$ holds.

Note that it is easy to verify that

$$
\begin{aligned}
w_{e}\left(\alpha_{e}\right) & =w_{e}\left(u_{1 e}, u_{2 e}, \ldots, u_{n}(e) e, u_{I}(e+1)\left(\alpha_{e}\right)\right. \\
& =\alpha_{e n}^{-I}(e)\left(\alpha_{e}\right) w_{e}\left(u_{1 e}, u_{2 e}, \ldots, u_{n}(e) e^{\left(\alpha_{e}\right)\left(L_{e} u_{I}(e+1)\right.}\right)\left(\alpha_{e}\right), e=1,2, \ldots, m-1 .
\end{aligned}
$$

$\operatorname{CASE}(i i)$. If $\left(L_{e} u_{s_{e}}(e+1)\right)\left(\alpha_{e}\right)=0, e=1,2, \ldots, m-1$ we let $c_{s_{e}}(e+1)=1$ and the rest coefficients zero. We then work as in Case (i). Otherwise we write (2.5) as

$$
\begin{aligned}
& \sum_{j=1}^{n(e)} c_{e j} u_{e j}{ }^{(k)}\left(\alpha_{e}\right)-c_{l n(e+1)} u_{\ln (e+1)}\left(\alpha_{e}\right)=\sum_{j=1}^{\bar{n}(e+1)} c_{j n(e+1)} u_{j n(e+1)}\left(\alpha_{e}\right), \\
& e=1,2, \ldots, m-1, k=0,1, \ldots, n(1) \text {. }
\end{aligned}
$$

Note now, that the rank of the coefficients matrix on the left hand side is $(n(1)+1)$ and thus we have a unique solution for the coefficients on the left hand side for any choice of the coefficients on the right hand side and for any $\alpha_{e} \in I$, $\mathrm{e}=1,2, \ldots, \mathrm{m}-1$.

The next theorem characterizes the conditions with the coefficients in (2.2) must satisfy in order that multiple branching can occur at $\left(\alpha_{1}, \alpha_{2}, \ldots, \alpha_{m-1}\right)$ with $\alpha_{e} \leq \alpha_{e+1}, e=1, \ldots, m-2$ and $\alpha_{e} \in I$.

THEOREM 2. The following are equivalent:

$$
\begin{gathered}
u \in N(M) \text { on }[c, d] \subset I \text { and } u \text { is as in }(2.2) . \\
\left(L_{e}\left(\sum_{j=1}^{n(e+1)} c_{e+1 j} u_{e+1 j}\right)\right)\left(\alpha_{e}\right)=0, e=1, \ldots, m-2 . \\
D^{k} e\left(L_{e+1}\left[\sum_{j=1}^{n(e)} c_{e j} u_{e j}\right]\right)\left(\alpha_{e}\right)=0, k_{e}=0,1, \ldots, n(e+1)-n(e)
\end{gathered}
$$


In particular, (2.8) with $c_{e+l j} \neq 0$ for at least one $u_{e+l j} \in E_{j}$ and (2.9) with $c_{e j} \neq 0$ for at least on $u_{e j} \in B_{e}-E_{e+1}$ are both necessary and sufficient conditions for $U \in N(M)$ to have a multiple branching at $\left(\alpha_{1}, a_{2}, \ldots, \alpha_{m-1}\right)$ on $[c, d]$.

PROOF. If $B_{e} \cap E_{e+1} \neq 0, e=1,2, \ldots, m-2$ the result is trivially true. Otherwise as in Theorem 1 , we have that $u \in N(M)$ if and only if

$$
\begin{aligned}
\sum_{j=1}^{n(e)} c_{e j} u_{e j}^{(k)}\left(a_{e}\right)=\sum_{j=1}^{n(e+1)} c_{e+1 j^{u}}^{u}(k)\left(a_{e}\right), k & =0,1, \ldots, n(1) \\
e & =1,2, \ldots, m-1 .
\end{aligned}
$$

The above can be written in the form

$$
\begin{aligned}
& \sum_{j=1}^{n(e)} c_{e j}^{\prime} u_{e j}^{(k)}\left(\alpha_{e}\right)=\sum_{j=1}^{n(e+1)} c_{e+1 j^{u}} u_{e+1 j}{ }^{(k)}\left(\alpha_{e}\right), \\
& k=0,1, \ldots, n(1), \\
& e=1,2, \ldots, m-1
\end{aligned}
$$

where $\left\{u_{e+1 j}\right\}_{j=1}^{\bar{n}(e+1)}=E_{e+1}$ and at least one $c_{e+1 j} \neq 0$. Here $c_{e j}^{\prime}=c_{e j}-c_{e+1 j}$ if $u_{e+l j} \in B_{e} \cap E_{e+1}, c_{e j}^{\prime}=c_{e j}$ otherwise. can be written

Now set $c_{e}^{\prime}(n(e)+1)=-1$ and $u_{e(n(e)+1)}(x)=\sum_{j=1}^{\bar{n}(e+1)} c_{e+1 j^{u} e+1 j}(k)(x)$ and (2.10)

$$
\sum_{j=1}^{n(e)+1} c_{e j}^{\prime} u_{e j}^{(k)}\left(a_{e}\right)=0, k=0,1, \ldots, n(l), e=1,2, \ldots, m-1 .
$$

Now, (2.11) has a nontrivial solution for $c_{e j}^{\prime}$ if and only if

but

$$
w_{e}\left(u_{1 i}, u_{2 i}, \ldots, u_{n(e) i}, u_{n}(e)+l i\right)\left(\alpha_{e}\right)=0,
$$

$$
\begin{aligned}
w_{e}\left(u_{1 i}, u_{2 i}, \ldots, u_{n(e) i}, u_{n}(e)+l i\right. & \left(\alpha_{e}\right) \\
& =a_{e n(e)}^{-1}\left(\alpha_{e}\right) w_{e}\left(u_{1 i}, u_{2 i}, \ldots, u_{n}(e) i\right. \\
& \left(\alpha_{e}\right) L_{e} u_{e}(n(e)+1)\left(\alpha_{e}\right),
\end{aligned}
$$

i.e., if and only if (2.8) holds and at least one $c_{p+l j} \neq 0$.

On the other hand, $u$ has a nontrivial branching at $\left(\alpha_{1}, \ldots, \alpha_{m-1}\right)$ if and only if (2.10) has a nontrivial solution for the coefficients on the right hand side. As before we set $c_{e}(\bar{n}(e+1)+1)=-1$ and

$$
u_{e}\left(\bar{n}(e+1)+1(x)=\sum_{j=1}^{n(e)} c_{e j}^{\prime} u_{e j}^{(k)}(x)\right.
$$

and (2.10) can now be written as

$$
\sum_{j=1}^{\bar{n}(e+1)+1} c_{e+1 j^{u} u+1 j}^{(k)}\left(\alpha_{e}\right)=0, k=0,1,2, \ldots, n(1), e=1, \ldots, m-1
$$

or

$$
A_{e} \bar{d}_{e}=\overline{0}
$$

in matrix form, where $A_{e}$ is the coefficient matrix in (2.12) and $\bar{d}_{e}$ the unknown vector. There will exist a nontrivial solution $\bar{d}_{e} \neq \overline{0}, e=1,2, \ldots, m-1$ if and only if the rank of $A_{e}, e=1,2, \ldots, m-1 \leq \bar{n}(e+1)$. But the $\bar{n}(e+1) \times \bar{n}(e+1)$ principle submatrix of $A_{e}$ is the Wronskian matrix evaluated at $\alpha_{e}$. Hence the rank of $A_{e} \geq \bar{n}(e+1)$. Therefore (2.13) will have a nontrivial solution if and only if the rank 
of $A_{e}$ is $\bar{n}(e+l)$. Now elementary row operations on $A_{e}$ show that this is equivalent to $(2.9)$.

We now show that $N(M)$ may contain infinitely many linearly independent functions.

THEOREM 3. Assume that either Case (i) holds in theorem for infinitely many $\left(\alpha_{1 i}, \alpha_{2 i}, \ldots, \alpha_{m-1 i}\right), i=1,2, \ldots$ or Case $(i i)$ holds. In either case, there is a sequence $\left\{u_{\alpha_{1 i} \alpha_{2 i} \ldots \alpha_{m-1 i}}\right\}_{i=1}^{\infty} \subset N(M)$ such that $u_{\alpha_{1 i} \alpha_{2 i} \ldots \alpha_{m-1 i}}$ has a multiple branching at $\left(\alpha_{1 i}, \alpha_{2 i} \ldots \alpha_{m-1 i}\right)$ with $\alpha_{e i}<a_{e+l i}, e=1, \ldots, m-2, i=1,2, \ldots$ and the set $\left\{u_{a_{1 i} a_{2 i} \ldots a_{m-1 i}}\right\}_{i=1}^{n}$ is linearly independent on $I$ for every $n$.

PROOF. We proceed by induction. We may assume without loss of generality that $a_{e i}<\alpha_{e i+1}, i=1,2, \ldots, e=1,2, \ldots, m-2$. Choose $u_{e+1 j}$ 's $\subset E_{e+1}$ then

$$
L_{p}\left(\sum_{j=1}^{\bar{n}(e+1)} c_{e+1 j} u_{e+1 j}\right)(x) \neq 0, x \in\left[\alpha_{e]}, \alpha_{(e+1)}\right] \text {. }
$$

Hence $u_{\alpha_{e l}}(x) \neq 0$ on $\left[\alpha_{e l}, \alpha_{(e+l) I}\right]$, so

on $I=[a, b]$.

$$
u_{\alpha_{11} \alpha_{21}} \ldots \alpha_{m-11}(x) \neq 0
$$

Now suppose that $u_{\alpha_{1 i} \alpha_{2 i} \ldots \alpha_{m-1 i}}, i=1,2, \ldots, n$ are linearly independent.

Suppose that there exist constants $a_{k}, i=1,2, \ldots, n+1$ :

$$
\sum_{i=1}^{n+1} \alpha_{i} u_{\alpha_{1 i} \alpha_{2 i}} \cdots \alpha_{m-1}(x)=0
$$

if $d_{n+1}=0$ then $d_{i}=0, i=1,2, \ldots, n$ and $\left\{u_{\alpha_{1 i} \alpha_{2 i} \ldots \alpha_{m-1}}\right\}_{i=1}^{n+1}$ is linearly independent. If $d_{n+1} \neq 0$

$$
u_{\alpha_{1 n+1}} \alpha_{2 n+1} \cdots \alpha_{m-1 n+1}(x)=\alpha_{n+1}^{-1} \sum_{i=1}^{n} \alpha_{i} u_{\alpha_{1 i} \alpha_{2 i}} \ldots \alpha_{m-1 i}
$$

for all $x \in I$ in particular for each $x \in\left(a_{e-l i}, \alpha_{e+l i}\right)$, but

whereas

$$
L_{e} u_{\alpha_{e n+1}}(x)=0, x \in\left(\alpha_{e n-p^{\alpha}+1}\right)
$$

$$
\left(d_{n+1}^{-1} \sum_{i=1}^{n} d_{i} u_{e i}\right) \in \operatorname{span} E_{e+1}
$$

when $x \in\left(\alpha_{e n}, \alpha_{e n+1}\right)$, so $L_{e} u_{\alpha_{e n+1}}(x) \neq 0$ for some $x \in\left(\alpha_{e n}, \alpha_{e n+1}\right)$ a contradiction. DEFINITION 2. Define the set $S_{i}$ by setting

$$
S_{i}=\left\{x \in I /\left(L_{i} u\right)(x)=0\right\} \text {. }
$$

Then since $L_{i} u, i=1,2, \ldots, m$ are continuous functions on $I$ the $S_{i}$ 's, $i=1,2,3, \ldots, m$ are closed sets and $S_{1} \cup S_{2} \cup \ldots U S_{m}=I$. In particular, any point $\alpha_{e} \in\left[\alpha_{e-1}, \alpha_{e+1}\right]$ at which an ordinary branching occurs on $\left[\alpha_{e-1}, \alpha_{e+1}\right], e=1,2, \ldots, m-1$ must belong to $\mathrm{S}_{\mathrm{e}-1} \cap_{\mathrm{e}}$ together with any limit point of the set of points at which ordinary branching occurs since $\mathrm{S}_{\mathrm{e}-1} \cap \mathrm{S}_{\mathrm{e}+1}$ is closed.

We show that $s_{e-1} \cap s_{e+1}$ is nowhere dense in $\left[\alpha_{e-1}, \alpha_{e+1}\right], e=1,2, \ldots, m-1$.

THEOREM 4. Assume that $u \in N(M)$ as in (2.2) and $B_{e} \cap E_{e+l}=\emptyset$. Then $\mathrm{s}_{\mathrm{e}-1} \cap \mathrm{s}_{\mathrm{e}+1}$ is nowhere dense in $\left[\alpha_{e-1}, \alpha_{e+1}\right], e=1,2, \ldots, m-1$. 
PROOF. Suppose that $\mathrm{S}_{\mathrm{e}-1} \cap \mathrm{S}_{\mathrm{e}+1}, \mathrm{e}=1,2, \ldots, \mathrm{m}-1$ contains a maximal closed interval $\left[\alpha_{e-1}^{\prime}, \alpha_{e+1}^{\prime}\right]$ with $\left|\alpha_{e+1}^{\prime}-\alpha_{e-1}^{\prime}\right| \neq 0, e=1,2, \ldots, m-1$. Then

$$
u(x)=\sum_{j=1}^{n(e+1)} c_{e j} u_{e j} \text { for } x \in\left[\alpha_{e-1}^{\prime}, \alpha_{e+1}^{\prime}\right] .
$$

Now let $\left(\alpha_{e-1}^{\prime \prime}, \alpha_{e+1}^{\prime \prime}\right) \subset\left[\alpha_{e-1}^{\prime}, \alpha_{e+1}^{\prime}\right]$. Then by Case $(i)$ in Theorem 1 there exist constants $c_{e j}^{(1)}, c_{e j}^{(2)}$, such that

$$
u\left(\alpha_{e-1}^{\prime \prime}, \alpha_{e+1 j}^{\prime \prime} x\right)= \begin{cases}\sum_{j=1}^{n(e)} c_{e j}^{(1)} u_{e j}(x) & \alpha_{e-1} \leq x \leq \alpha_{e-1}^{\prime \prime} \\ n(e+1) & \alpha_{e-1}^{\prime \prime} \leq x \leq \alpha_{e+1}^{\prime \prime} \\ \sum_{j=1}^{n} c_{e j} u_{e j}(x) & \alpha_{e+1}^{\prime \prime} \leq x \leq \alpha_{e+1} \\ \sum_{j=1}^{n(e)} c_{e j}^{(2)} u_{e j}(x) & \end{cases}
$$

belongs to $N(M)$ since

$$
L_{e}\left(\sum_{j=1}^{n(e+1)} c_{e j} u_{e j}\right)(z)=0
$$

at $z=\alpha_{e-1}^{\prime \prime}, \alpha_{e+1}^{\prime \prime} \cdot$ But

$$
L_{e}\left(\sum_{j=I}^{n(e+I)} c_{e j} u_{e j}\right)(x)=0
$$

on $\left[\alpha_{e-1}^{\prime \prime}, \alpha_{e+1}^{\prime \prime}\right]$. Hence $U\left(\alpha_{e-1}^{\prime \prime}, \alpha_{e+1 j}^{\prime \prime} x\right) \in N\left(L_{e}\right)$. Since $\sum_{j=1}^{n(e+1)} c_{e j}^{u} u_{e j}(x) \in N\left(L_{e}\right)$, $e=1,2, \ldots, m-1$ the proof of Theorem 3 shows that the set $B_{e} U\left\{u\left(\alpha_{e-1}^{\prime \prime}, \alpha_{e+1}^{\prime \prime} j^{x}\right)\right\}$ is linearly independent. But this contradicts $a\left(L_{e}\right)=n(e), e=1,2, \ldots, m$.

We now assume that $n(l)=N(2)=\ldots=n(m)$ for simplicity (the other cases can be dealt analogously) and consider the following problem: given $\left(z_{0}, z_{1}, \ldots, z_{n}(1)-1\right) \in \mathbb{R}^{n(1)}$ and $z \in I$ find $u$ such that

$$
\begin{gathered}
M_{u}=\left(L_{1} u\right)\left(I_{2} u\right) \ldots\left(I_{m} u\right)=0 \\
D^{n(I)} u(z)=z_{i}, i=0, I, \ldots, n(I)-I .
\end{gathered}
$$

if $\mathrm{N}\left(\mathrm{L}_{\mathrm{e}}\right) \neq \mathrm{N}\left(\mathrm{L}_{\mathrm{e}+1}\right), \mathrm{e}=1,2, \ldots, \mathrm{m}-1$, then we have at least $\mathrm{m}$ solutions, the unique solutions belonging to $N\left(L_{e}\right), e=1,2, \ldots, m-1$. In addition according to Theorems 1 and 2 we may have solutions with one or many multiple ordinary branchings.

In the event that $L_{e}, e=1,2, \ldots, m$ have constant coefficients we proceed as follows: - let $s_{j e}, j=1,2, \ldots, n(1), e=1,2, \ldots, m$ denote the solutions of the characteristic equation $L_{e}$ and assume $u \in N\left(L_{e}\right)$ on some subinterval $I(z)$ of $I$ containing $z$, then the restriction $\bar{u}$ of $u$ on $I(z)$ can be written

$$
\bar{u}(x)=\sum_{j=1}^{n(1)} c_{j e} e^{s j e^{x}}
$$

where $\left\{e^{s} j e^{x}\right\}_{j=1}^{n(I)}$ spans $N\left(L_{e}\right)$ and $c_{j e}$ are uniquely determined by (2.14). By (2.9) we must have

$$
L_{e+1}\left(\bar{u}\left(\alpha_{e}\right)\right)=0, \quad e=1,2, \ldots, m-1 .
$$

It follows that 
where

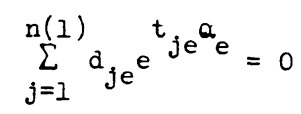

$$
\begin{gathered}
d_{j e}=c_{j e} \sum_{i=0}^{n(1)} c_{i e+1} t_{i e}^{i}, i=1,2, \ldots, n(1) \\
t_{i e}=s_{i e}-s_{n}(1) e, e=1,2, \ldots, m .
\end{gathered}
$$

Note that each one of the equations in (2.15) can have at most $n(1)-1$ real solutions if the $a_{j e}$ 's and $t_{j e}$ 's are all real [7].

Denote by $\alpha_{\mathrm{pl}}, \alpha_{\mathrm{p} 2}, \ldots, \alpha_{\mathrm{pn}(1)-1}$ the solutions obtained in the $\mathrm{p}=$ equation in (2.15), $\mathrm{p}=1,2, \ldots, \mathrm{m}-1$ and assume that (the other cases can be dealt analogously)

$$
\begin{aligned}
\alpha_{11} & \leq \alpha_{12} \leq \alpha_{13} \leq \ldots \leq \alpha_{1 n(1)-1} \leq \\
\alpha_{21} & \leq \alpha_{22} \leq \alpha_{23} \leq \ldots \leq \alpha_{2 n(1)-1} \leq \\
& \vdots \alpha_{m-11} \leq \alpha_{m-13} \leq \ldots \leq \alpha_{m-1 n(1)-1}
\end{aligned}
$$

Inequality (2.18) shows that we can have at most $(n(I)-1)^{m-1}$ ordinary multiple branchings, e.g. $\left(\alpha_{11}, \alpha_{21}, \ldots, \alpha_{m-11}\right)$ is one of them. We have thus proved.

THEOREM 5. If $L_{i}, i=1,2, \ldots, m$ have constant coefficients, then there exists a solution $u \in N(M)$ ( $u$ as in (2.2)) to the intial value problem (2.14) having a multiple ordinary branching $\left(\alpha_{1}, \alpha_{2}, \ldots, \alpha_{m-1}\right)$ with $\alpha_{e} \in I, e=1,2, \ldots, m-1$ if and only if $\alpha_{e}$ is a root of the exponential polynomial (2.15), where the $\alpha_{j e}$ 's and $t_{j e}$ 's are all real and they are given by (2.16) and (2.17).

Moreover if $(2.18)$ holds there are at most $(n(1)-1)^{m-1}$ solutions $u \in N(M)$ (u as in (2.2)).

THEOREM 6. Assume that the hypotheses of Theorem 5 are satisfied. Then there are at most

$$
(m-1)(n(1)-1)(n(1)-2)^{n-1}
$$

solutions $u$ ( $u$ as in (2.2)) to the initial value problem having exactly $n$ multiple branchings $\left(\alpha_{1}, \alpha_{2}, \ldots, \alpha_{m-1}\right)$ in $I$ where any $m-2$ of the $\alpha_{e}$ 's are fixed $\mathrm{e}=1,2, \ldots, \mathrm{m}-1$.

Moreover in this case if there are no solutions with $n+l$ multiple branchings then the total number of solutions to the problem

$$
\mathrm{Mu}=0
$$

is bounded by

$$
(m-1)(n(1)-1) \sum_{j=0}^{n-1}(n(1)-2)^{j} \text {. }
$$

PROOF. Without loss of generality we can assume that $\alpha_{11}$ denote the first point at which a branching occurs and $u \in N\left(L_{1}\right)$ on some subinterval $I(z)=\left[z, \alpha_{11}\right]$. Then $u \in N\left(I_{2}\right)$ on $\left[\alpha_{11}, \alpha_{11}+c\right]$, for some $\varepsilon>0$. There are at most m-l possible values for $\alpha_{11}$. Suppose $w>\alpha_{11}$ is the next point at which a multiple branching of $u$ occurs. Then $u \in N\left(L_{2}\right)$ on $\left[\alpha_{11}, w\right]$. Hence there exist uniquely determined $c_{j 2}\left(\alpha_{11}\right), j=1,2, \ldots, n(1)$ such that 


$$
u(x)=\sum_{j=1}^{n(1)} d_{j 2}\left(\alpha_{11}\right) u_{j 2}(x)
$$

on $\left[\alpha_{11}, w\right]$ where $\left\{u_{j 2}\right\}_{j=I}^{n(I)}$ span $N\left(L_{2}\right)$. By Theorem 2,

$$
\left[L_{1}\left(\sum_{j=1}^{n(1)} d_{j}\left(a_{11}\right) u_{j 2}\right)\right](v)=0
$$

at $v=a_{11}$ and $v=w$. Hence there are $m-2$ possible w's with $w>a_{11}$. This argument applies again for the next branching. Since this argument can be applied in any of the $m-1$ rows in (2.18), this proves (2.19).

Finally (2.20) can easily be proved if we use (2.19) for $j=0,1,2, \ldots, n$ and add the results.

REMARK 1. (a) We can assume in Theorem 6 that any $h$ points $h \in\{1,2, \ldots, m-1\}$ are fixed from $\left(\alpha_{1}, \alpha_{2}, \ldots, \alpha_{m-1}\right)$ then proceeding as in Theorem 6 we can prove that the corresponding relations for (2.19) and (2.20) are respectively

$$
(m-1-(h-1))(m(1)-1)^{h}(n(I)-2)^{h-1}
$$

and

$$
(m-1-(h-1))(n(1)-1)^{h^{n}-1}(n(1)-2)^{h}
$$

(b) Up till now we obtained the cardinality results in Theorems 5, 6 and in (a) above by assuming that (2.18) is true and $u$ as in (2.2). But (2.2) can be written in $(m-1) !$ different ways by interchanging the role of the $L_{i}{ }^{\prime} s, i=1,2, \ldots, m$. Therefore in general all the cardinality results obtained up till now can be multiplied by $(\mathrm{m}-1)$ !

(c) If the $L_{i}, i=1,2, \ldots, m$ are nonconstant but continuous (as in the Introduction) we can restate Theorem 5 and (2.2). However the conclusions and the proofs are going to be exactly analogous.

We now provide examples for Theorems 4 and 6 and (1.4).

3. APPLICATIONS.

EXAMPLE 1 . Let $m=2$ and consider the function $f$ defined by

or

$$
f(x)= \begin{cases}x^{8} \ln \frac{1}{x^{2}}, & x \neq 0 \\ 0, & x=0\end{cases}
$$

$$
u_{1}(x)=e^{f(x)}, u_{2}(x)=e^{2 f(x)}, L_{1} u=u^{\prime}-f^{\prime}(x) u, L_{2} u=u^{\prime}-2 f^{\prime}(x) u .
$$

Then $u_{1} \in N\left(L_{1}\right), u_{2} \in N\left(L_{2}\right)$ and a $u \in N(M)$ can be written as

$$
u(x)=\left\{\begin{array}{ll}
c e^{2 f(x)}, & -6 \leq x \leq 0 \\
d e^{f(x)}, & 0 \leq x \leq \varepsilon
\end{array}, \epsilon>0\right.
$$

$$
u(x)=\left\{\begin{array}{ll}
c e^{f(x)}, & -\epsilon \leq x \leq 0 \\
d e^{2 F(x)}, & 0 \leq x \leq \varepsilon
\end{array}, \epsilon>0\right.
$$

That is, 0 is a limit point of branching points of $u$. 
In the event that the characteristic equations of $L_{i}, i=1,2, \ldots, m$ have complex roots (2.15) may have infinite solutions to the initial value problem on $(-\infty, \infty)$ even if we have one ordinary multiple branching in $(-\infty, \infty)$.

EXAMPLE 2. Let $m=2, L_{1}=D^{2}+1, L_{2}=D^{2}+4, u(0)=0, u^{\prime}(0)=1$. Let $u \in \mathbb{N}\left(L_{1}\right)$ on $[-\epsilon, \varepsilon]$ for some $c>0$. Then

$$
u_{1}(x)=-\frac{i}{2} e^{i x}+\frac{i}{2} e^{-i x}=\sin x
$$

and $(2.15)$ due to $(2.16)$ and $(2.17)$ becomes

$$
e^{2 i \alpha}=1
$$

therefore $\alpha_{n}=n \pi, n=0,1,2, \ldots$

$$
u_{2}(x)=\frac{1}{4 i} e^{2 i x}-\frac{1}{4 i} e^{-2 i x}=\frac{1}{2} \sin 2 x \text {, etc. }
$$

EXAMPLE 3. Consider the equation

$$
\frac{d M}{d x}-\lambda M=0 ? .
$$

Let $L_{1}=(D-1)(D-2)(D-3), L_{2}=(D-4)(D-5)(D-6), L_{3}=(D-7)(D-8)(D-9)$ and $\lambda=0$. Then

and

$$
\begin{aligned}
& u_{1}(x)=2 e^{x}-3 e^{2 x}+e^{3 x} \\
& u_{2}(x)=5 e^{4 x}-9 e^{5 x}+4 e^{6 x} \\
& u_{3}(x)=8 e^{7 x}-15 e^{8 x}+7 e^{9 x}
\end{aligned}
$$

$$
\begin{aligned}
& \mathrm{L}_{2} \mathrm{u}_{1}(x)=0 \rightarrow-120 e^{x}+72 e^{2 x}-6 e^{3 x}=0 \rightarrow \alpha=\ln 2, \ln 10 \\
& \mathrm{~L}_{1} \mathrm{u}_{2}(x)=0 \rightarrow 30 e^{4 x}-216 e^{5 x}+240 e^{6 x}=0 \rightarrow \alpha=\ln \left(\frac{108+\sqrt{4464}}{240}\right) \\
& \mathrm{L}_{3} \mathrm{u}_{2}(x)=0 \rightarrow-300 e^{4 x}+216 e^{5 x}-24 e^{6 x}=0 \rightarrow a=\ln \left(\frac{54 \pm \sqrt{1116}}{12}\right) \\
& \mathrm{L}_{2} \mathrm{u}_{3}(x)=0 \rightarrow 48 e^{7 x}-360 e^{8 x}+420 e^{9 x}=0 \rightarrow \alpha=\ln \left(\frac{90 \pm \sqrt{3060}}{210}\right)
\end{aligned}
$$

So we can have multiple branchings at

$$
\begin{aligned}
& \left(\ln 2, \ln \left(\frac{54+\sqrt{1116}}{12}\right)\right), \\
& \left(\ln \left(\frac{108+\sqrt{4464}}{240}\right), \ln \left(\frac{54+\sqrt{1116}}{12}\right)\right), \\
& \left(\ln \left(\frac{108+\sqrt{4464}}{240}\right), \ln \left(\frac{54-\sqrt{1116}}{12}\right)\right), \\
& \left(\ln \left(\frac{108-\sqrt{4464}}{240}\right), \ln \left(\frac{54+\sqrt{1116}}{12}\right)\right), \\
& \left(\ln \left(\frac{108-\sqrt{4464}}{240}\right), \ln \left(\frac{54-\sqrt{1116}}{12}\right)\right),
\end{aligned}
$$

and

$$
\left(\ln \left(\frac{108-\sqrt{4464}}{240}\right), \ln \left(\frac{90+\sqrt{3060}}{210}\right)\right) \text {. }
$$

For example we can have the solution $u \in N(M)$ given by 


$$
u(x)= \begin{cases}2 e^{x}-3 e^{2 x}+e^{3 x}, & -\infty<x \leq \ln 2 \\ 5 e^{4 x}-9 e^{5 x}+4 e^{6 x}, & \ln 2 \leq x \leq \ln \left(\frac{54+\sqrt{1116}}{12}\right) \\ 8 e^{7 x}-15 e^{8 x}+7 e^{9 x}, & \ln \left(\frac{54+\sqrt{1116}}{12}\right) \leq x<+\infty,\end{cases}
$$

etc.

The above are solutions corresponding to the order $\left(\mathrm{L}_{1}, \mathrm{~L}_{2}, \mathrm{~L}_{3}\right)$. But we can obtain additional solutions corresponding to $\left(\mathrm{L}_{1}, \mathrm{~L}_{3}, \mathrm{~L}_{2}\right),\left(\mathrm{L}_{2}, \mathrm{~L}_{1}, \mathrm{~L}_{3}\right),\left(\mathrm{L}_{2}, \mathrm{~L}_{3}, \mathrm{~L}_{1}\right)$, $\left(L_{3}, L_{1}, L_{2}\right)$ and $\left(L_{3}, L_{2}, L_{1}\right)$.

\section{REFERENCES}

1. ALLGOWER, E.L. and PRENTER, P.M. On the Branching of Solutions of Quadratic Differential Equations, Aequationes Mathematicae 10 (1974), 81-96.

2. AMES, W. Ordinary Differential Equations in Transport Processes, Academic Press, New York, 1968.

3. CHOW, S.N. and HALE, J.K. Methods of Bifurcation Theory, Springer Verlag, New York, 1982.

4. DAVIS, H.T. Introduction to Nonlinear Differential and Integral Equations, Dover, New York, L962.

5. HARTMAN, P. Ordinary Differential Equations, Wiley, New York, 1964.

6. KAMKE, E. Differentialgleichungen Lösungs-Methoden und Lösungen, Chelsea, New York, 1959.

7. LANGER, R.E. On the Zeros of Exponential Sums and Integrals, Bull. Amer. Math. Soc. 37 (1931), 213-239. 


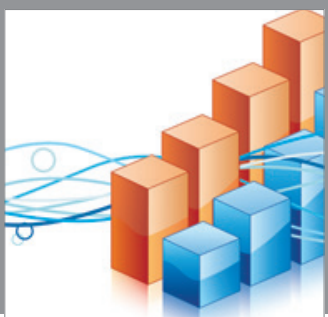

Advances in

Operations Research

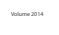

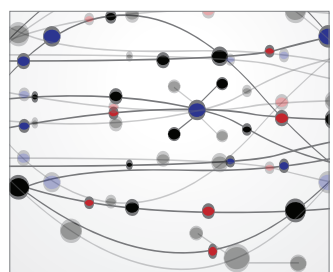

\section{The Scientific} World Journal
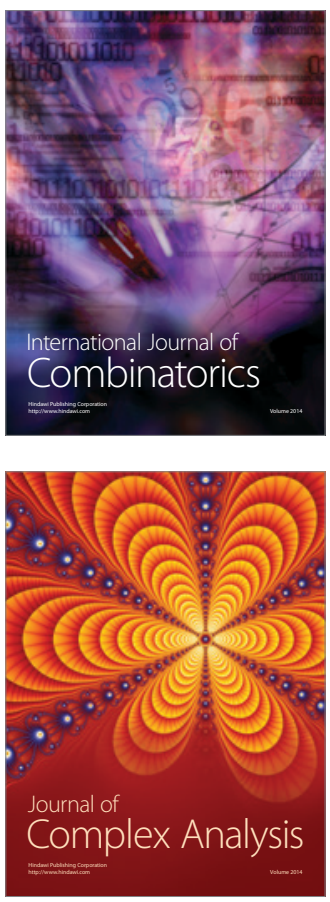

International Journal of

Mathematics and

Mathematical

Sciences
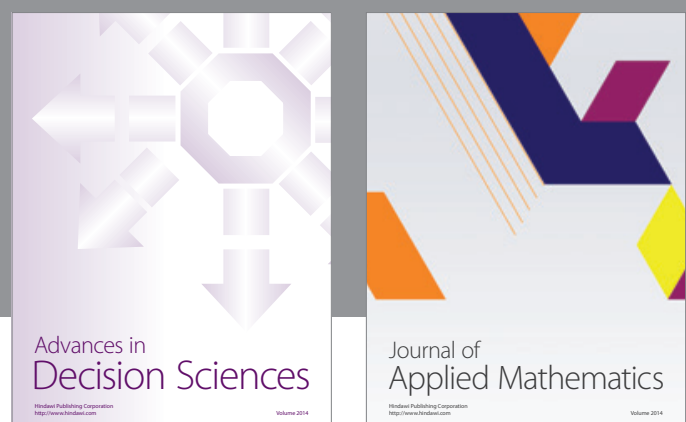

Journal of

Applied Mathematics
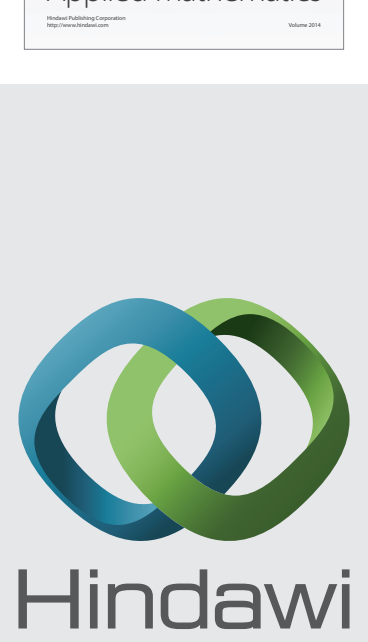

Submit your manuscripts at http://www.hindawi.com
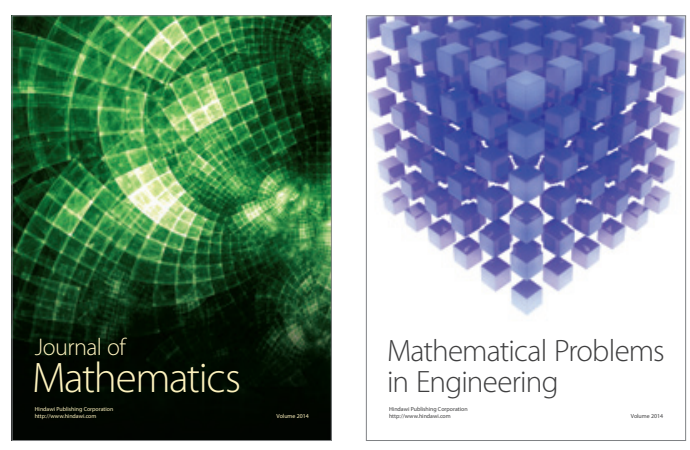

Mathematical Problems in Engineering
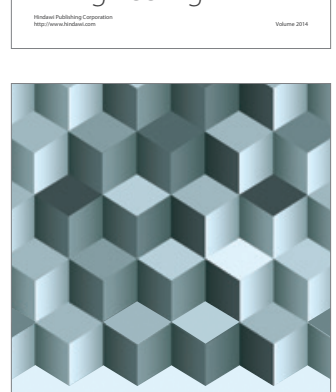

Journal of

Function Spaces
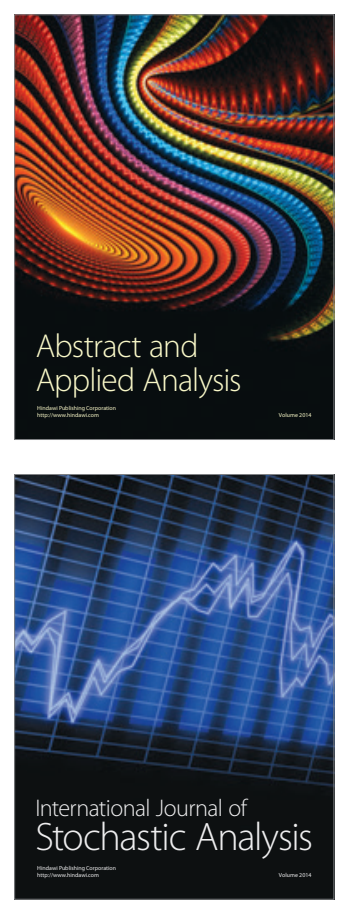

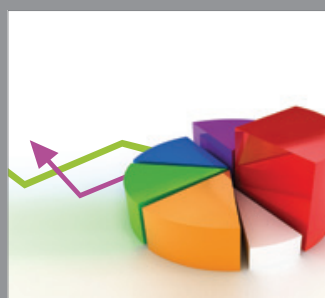

ournal of

Probability and Statistics

Promensencen
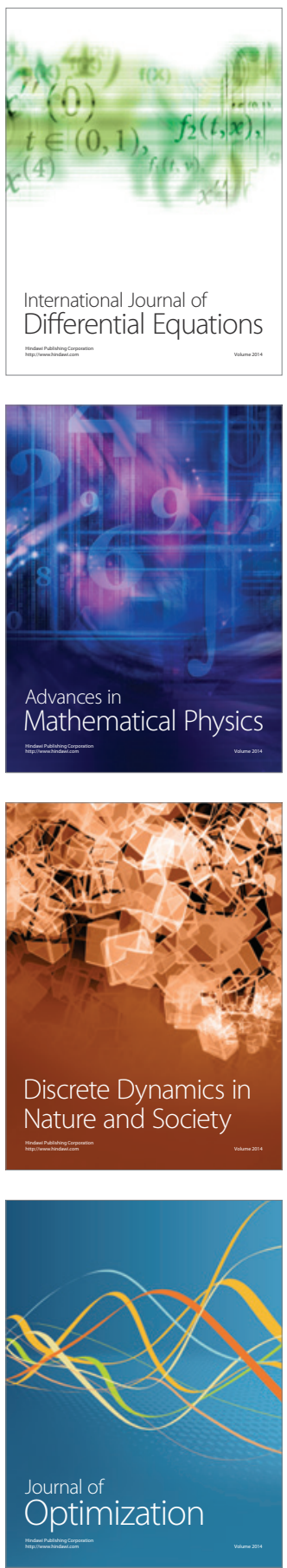